\title{
Transport and Deposition of Wear Particles in Computer Disk Drives
}

\author{
Chuen-Jinn Tsai ${ }^{*}$ David Y. H. Pui **, Benjamin Y. H. Liu **
}

(Received: 24 October 1990; resubmitted: 15 April 1991)

\begin{abstract}
To understand the roles that wear particles play in disk crashes, analytical and numerical approaches have been used to study three dimensional fluid flow and wear particle transport and deposition in computer disk drives. The study shows that wear particles are flying very close to the disk surface. They can deposit on the disk surface and cause damage to disk drives.
\end{abstract}

Cooling air flow through the disk hub of corotating disks has been found to play important roles in removing wear particles from disk drives. Parametric study has shown that the optimum cooling air flow rate is 12 slpm for 8 " disk with the Reynolds number of 150,000 .

\section{Introduction}

Since the invention of the magnetic recording technology at the beginning of the twentieth century (Croll [1]), it has been playing an important role in the audio recording, video recording and in the computer industries. According to the Porter report [2], the annual world wide revenue of rigid magnetic disk drives alone will reach 27 billion dollars in 1990. This figure is expected to rise to 32 billion dollars in 1992.

The magnetic recording technology has been advanced from the earliest magnetic tape, to magnetic drum and finally, with the invention of the slider air-bearing in 1957 (Lesser and Haanstra [3]), the magnetic disk file was introduced by IBM. Twenty five years after the introduction of the first magnetic disk storage device, according to Croll [1], the areal density has been increased by a factor of 6000 . Today, the storage capacity of a magnetic disk drive as high as several hundred megabytes, or even several gigabytes is not uncommon.

The secret of this several orders of magnitude increase in the storage density is due to the reductions in the head/disk spacing, head gap length and media thickness. Above all, the selfacting air-bearing, which has led to the reduction in the head/disk spacing, is one of the most important factor. Today, the typical flying height for a high performance, high capacity rigid disk drives has been reduced well under $0.254 \mu \mathrm{m}(10$ microinches). With the growing demand to the recording density, the flying height has to be even lower. According to the Porter report [2], $0.127 \mu \mathrm{m}$ ( 5 microinches) has been used to achieve 100 megabytes capacity in a single disk $3.5^{\prime \prime}$ drive. A commonly used taper-flat slider air-bearing (or read/write head) can be seen in Figure 1. It has an inlet taper to help fast

* C. J. Tsai, Institute of Environmental Engineering, National Chiao Tung University, Taiwan (Republic of China).

** Prof. D. Y. H. Pui (to whom all correspondence should be sent), Prof. B. Y. H. Liu, Particle Technology Laboratory, Mechanical Engineering Department, University of Minnesota, Minneapolis, Minnesota 55455 (USA).

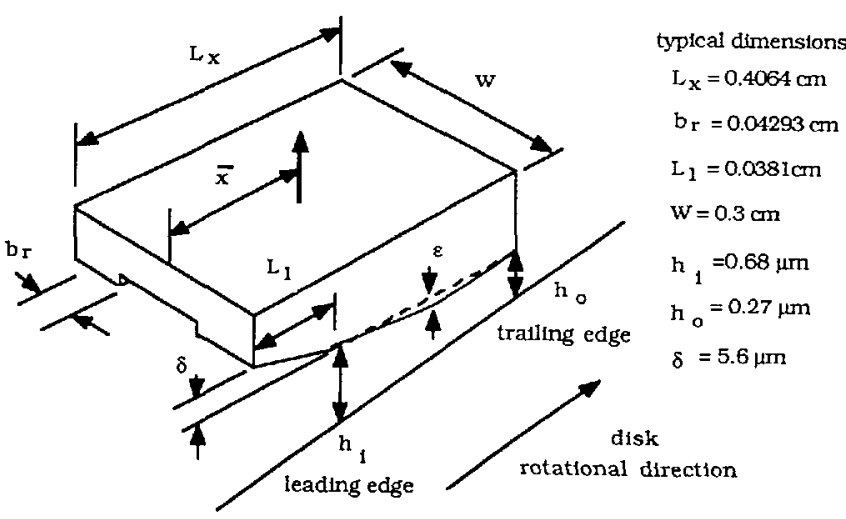

Fig. 1: A typical taper-flat slider air-bearing (read/write head).

take-off and increase leading edge stiffness. The following flat region is the main part of the air-bearing. The magnetic coils and core are deposited on the trailing edge of the slider by thin film technology. The ratio of the leading film thickness to the trailing film thickness is typically around three. The flying height refers to the trailing film thickness.

The typical flying height of a slider is compared with the size of various particles in Figure 2. It is seen that the flying height is several orders of magnitude smaller than the size of particles that may be found on the disk surface. As a result of this extremely small flying height, foreign particles become more likely to cause temporary loss of memory or even permanent damage to the disk/head assembly as they intrudes the air-bearing. In addition, physical contact between the disk and slider is more likely. Wear particles can be generated when physical contact occurs. An experimental setup and the results of wear particle measurement in computer disk drives can be found in Tsai et al. [4].

The purpose of this paper is aimed at providing some answers to the following problems in regard to wear particles in computer disk drives. These problems have rarely been addressed in the open literature. They include: (1) the initial speed and direc- 


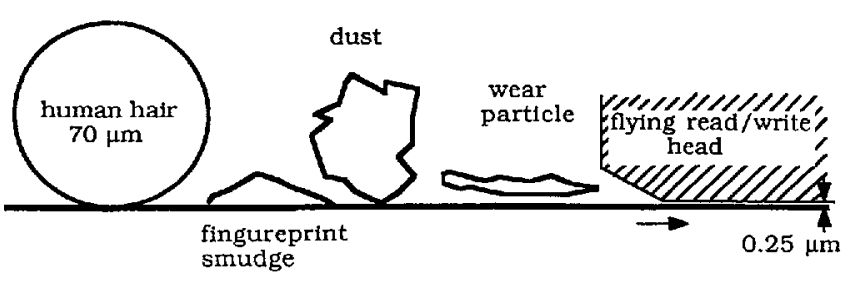

magnetic disk

Fig. 2: Comparison of the flying height of a slider with the size of other particles.

tion of a wear particle as it is emitted from the disk/head interface. (2) possibility of wear particle deposition on the disk surface and cause potential problem to the disk drive. (3) the influence of cooling air flow from the disk hub on wear particle transport. (4) effect of external forces on wear particle transport in disk drives.

Initial attempt is to calculate the initial wear particle direction and velocity as it is first generated. This is followed by the flow field and particle transport study. Cooling air flow from the disk hub of corotating disk enhances cooling effect significantly. Its influence on wear particle behavior is expected to be important and is also considered. To study wear particle transport, it is unreasonable to treat particles as a second phase since wear particle generation is intermittent and particles are very few in number. Instead, a single particle tracing method is more practical. This is presented next where a numerical method is used to determine particle transport and deposition taking into account various influencing factors such as electrostatic force and Brownian diffusion.

\section{The Most Probable Wear Particle Initial Speed and Direction}

When a wear particle is generated as a result of the physical contact between the slider and disk, it is likely to be emitted randomly from the trailing edge of the slider because the flying height is the smallest and hence the physical contact is more susceptible there. It is essential to understand the possible initial particle emission speed and direction before tracing the final destiny of a wear particle is possible.

It has been shown by Tsai et al. [5], a minimum wear particle size exists in any sliding wear situation. Particles greater than the minimum size normally are not generated in only one asperity contact. Instead, they are generated due to void nucleation and crack propagation processes upon number of repeated loading and unloading cycles. At the moment when crack propagation is unstable and the crack is sheared to the surface, a wear particle is generated. Hence the surface energy increase and work done in plastic deformation at the crack tip can be neglected when estimating wear particle speed. Work done by shear force in the vertical $z$ direction can then be assumed to be converted entirely to the vertical kinetic energy $E_{\mathrm{kv}}$. According to Tsai et al. [5], the work done by friction force in vertical direction and hence $E_{\mathrm{kv}}$ of a flake particle (length $L \cdot$ width $b \cdot$ thickness $t$ ) can be calculated as

$E_{\mathrm{kv}}=\frac{1}{2} L b t \rho_{\mathrm{p}} V_{\mathrm{v}}^{2}=\frac{3 \mu_{\mathrm{f}}^{2} F_{\mathrm{r}}^{2} b^{2} L^{2}}{5 E A_{\mathrm{n}}^{2} t}$ where the right hand side represents the work done by friction force. Or in terms of the vertical velocity component $V_{\mathrm{v}}$

$V_{\mathrm{v}}=\sqrt{\frac{2}{\rho_{\mathrm{p}}}\left(\frac{3 \mu_{\mathrm{f}}^{2} F_{\mathrm{r}}^{2} b L}{5 E A_{\mathrm{n}}^{2} t^{2}}\right)}$.

In the above equations, $F_{\mathrm{r}}$ is the real contact load, $A_{\mathrm{n}}$ is the nominal contact area, $E$ is the Young's Modulus, $\mu_{\mathrm{f}}$ is the coefficient of friction. Also nominal contact stress is equal to $F_{\mathrm{r}} / A_{\mathrm{n}}$.

The horizontal velocity $V_{\mathrm{h}}$ due to friction force alone can be estimated from the equilibrium between friction force and fluid drag force as

$\mu_{f} \frac{F_{\mathrm{r}}}{A_{\mathrm{n}}} L b=\frac{6 \pi \mu b^{\prime} V_{\mathrm{h}} K^{\prime}}{C_{\mathrm{D}_{\mathrm{ev}}}}$.

When including the disk linear velocity $V_{\mathrm{d}}$, the horizontal velocity $V_{\mathrm{h}}$ can be calculated as

$V_{\mathrm{h}}=V_{\mathrm{d}}+\frac{\mu_{\mathrm{f}} F_{\mathrm{r}} C_{\mathrm{D}_{\mathrm{ev}}} \sqrt{L b}}{6 \pi^{3 / 2} A_{\mathrm{n}} \mu K^{\prime}}$, for particles from the head

$V_{\mathrm{h}}=V_{\mathrm{d}}-\frac{\mu_{\mathrm{f}} F_{\mathrm{r}} C_{\mathrm{D}_{\mathrm{ev}}} \sqrt{L b}}{6 \pi^{3 / 2} A_{\mathrm{n}} \mu K^{\prime}}$, for particles from the disk

In the above equations, $K^{\prime}$ is the shape factor for a flake moving perpendicular to the polar axis (refer to the Appendix $C$ in Tsai [5]), $C_{\mathrm{D}_{\mathrm{av}}}$ is the slip correction factor for equal volume diameter of the flake, which is equal to $[3 L b t /(4 \pi)]^{1 / 3}, \mu$ is the dynamic viscosity of air.

The predicted particle velocities can be seen in Figure 3 neglecting the linear disk velocity for the time being. The figure is plotted under the assumption that the length of the flake is equal to its width. It is seen that for the flake length from 0.05 to $5 \mu \mathrm{m}$, the particle vertical velocity component $(0.1$ to $10 \mathrm{~cm} / \mathrm{sec}$ ) is several orders of magnitude smaller than its horizontal counterpart ( 1.000 to $10.000 \mathrm{~cm} / \mathrm{sec}$ ) for both $t=0.1 \mu \mathrm{m}$ and $t=0.04 \mu \mathrm{m}$. This is because the vertical force to push particle away from the surface is usually very small compared to the horizontal force. The effect of friction force is to increase both velocity components as obvious in the previous equations. In Figure 3, the following parameters are used: $E=3.09 \times 10^{12}$ dyne $/ \mathrm{cm}^{2}, F_{\mathrm{r}}=15 \mathrm{gm}, A_{\mathrm{n}}=0_{\mathrm{g}}^{\mathrm{t}} 11 \mathrm{~cm}^{2}, \rho_{\mathrm{p}}=4.25 \mathrm{gm} / \mathrm{cm}^{3}$,

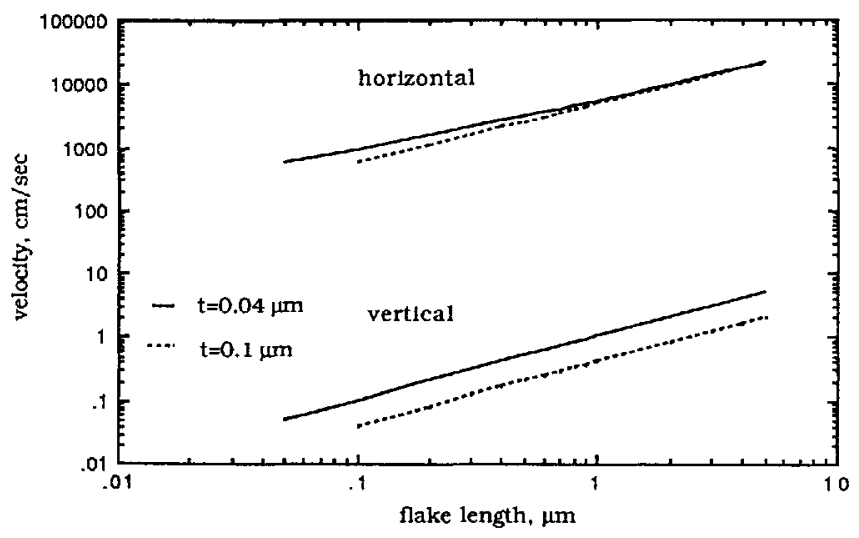

Fig. 3: Horizontal and vertical velocity components versus flake length in the drag-mode wear particle generation. 
$\mu=1.83 \times 10^{-4}$ dyne $\cdot \mathrm{sec} / \mathrm{cm}$. Furthermore, assume $L=b$, $\mu_{\mathrm{f}}=1.0$.

The above analysis assumed the particles were "dragged" out of the disk surface by friction force. In some cases, a potential wear particle may have its tail end curled over the disk surface and be "kicked" away in the subsequent asperity contact. Dynamic vibration of the slider may also impart additional vertical force to the potential wear particles. Under this conditions, the vertical velocity component is larger than what has been calculated here.

Using the calculated particle initial velocity, it is expected that wear particles will move very close to the disk surface since both the vertical velocity component and particle size are small. Figure 4 shows an example of the vertical stopping distance of a wear particle assuming it is emitting from the horizontal disk surface at $r=6.556 \mathrm{~cm}$ and the disk rotating speed is $3600 \mathrm{rpm}$. Instead of using the calculated values, which will indicate that particles are virtually flying on the disk surface, the initial wear particle speed is assumed to be the same as the disk linear speed and the emitting angle to be $\mathbf{4 5}$ degree from the disk surface. It is seen that the wear particles will be stopped very close to the disk surface initially and then transported from there. For example, the stopping distances are $1.6,18,63 \mu \mathrm{m}$ for $0.1,0.5$ and $1.0 \mu \mathrm{m}$ particles in aerodynamic diameter respectively.

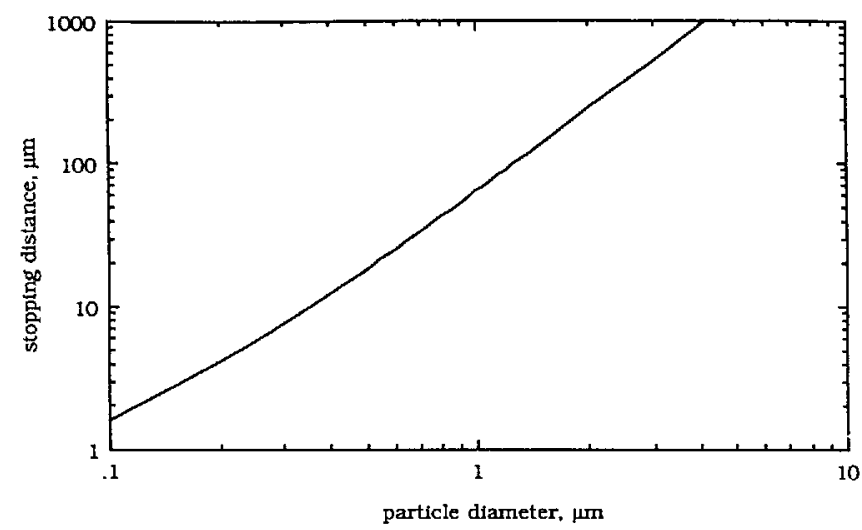

Fig. 4: Vertical stopping distance of a wear particle versus aerodynamic diameter.

To prove that wear particles are indeed flying very close to the disk surface, experiments were performed using two different sampling systems to sample wear particles from a single disk wear tester. Both systems used a condensation nucleus counter as the particle detecting instrument. In one of the sampling system, a scoop with open area $2 \mathrm{~cm}^{2}$ was placed $0.5 \mathrm{~cm}$ away from the trailing edge and its bottom surface was $20 \sim 30 \mu \mathrm{m}$ above the disk surface- a lowest possible position achievable in the experiment. The sampling flow rate was $20 \mathrm{slpm}$. In the other system, a high velocity nitrogen jet blowing at about $100 \mathrm{~m} / \mathrm{sec}$ at 20 degree to the disk surface was used. This was to blow wear particles away from the trailing edge. The nozzle was located $0.5 \mathrm{~cm}$ from the trailing edge of the slider and $0.2-0.3 \mathrm{~cm}$ from the disk surface. Initially, both systems were checked carefully making sure there were no background particles. The wear tester was undergoing CSS cycles (contact start and stop, 17 seconds/cycle).

The test results showed that nitrogen jet system has sampled wear particles effectively while sampling scoop system virtually has detected no particles. The nitrogen jet system detected very low particle concentration about $2.0 \mathrm{\#} / \mathrm{cm}^{3}$ from 0 to 2000 cycles. As wear proceeded, the concentration increased to $10 \# / \mathrm{cm}^{3}$ from $2000-6000$ cycles. Beyond 6000 cycles, the variation of the particle concentration was more random. In contrast, the sampling scoop system could not sample wear particles at all. It detected $1.0 \mathrm{\#} / \mathrm{cm}^{3}$ throughout the experiment despite an apparent wear track was observed.

These experimental results suggest that wear particles are indeed flying very close to the surface: They deposit on the disk surface easily and cause damage to the read/write head as it flies over the deposited particles. As one will see, the following numerical study on flow field and particle transport also reaches similar conclusion.

\section{Flow Field in Disk Drives}

\subsection{Review of the Flow Fields in Disk Drives}

The flow field in shrouded corotating disks is different from that in a single rotating disk. It has been numerically simulated by $A$ nanth [6] and experimentally visualized by $A$ brahamson et al. [7]. According to Abrahamson et al. [7], there are three basic regions between two corotating disks, namely, inner region, outer region and shroud boundary layer region. This is shown in Figure 5 for flow between two corotating disks with the following specific geometry and conditions-disk radius: $56 \mathrm{~cm}$, hub radius: $28 \mathrm{~cm}$, rotational speed: $25 \mathrm{rpm}$, diskshroud spacing: $3.14 \mathrm{~cm}$, disk-disk spacing: $2.8 \mathrm{~cm}$, disk thickness: $1.3 \mathrm{~cm}$, medium: water at $13{ }^{\circ} \mathrm{C}$. The inner region extends from the hub to about one third to one half of the radial distance between the hub and the shroud. It is two-dimensional, laminar (has very little mixing) and in solid body rotation with the disks. The shape of the inner region is polygonal with the number of sides determined by the Ekman number and diskdisk spacing.

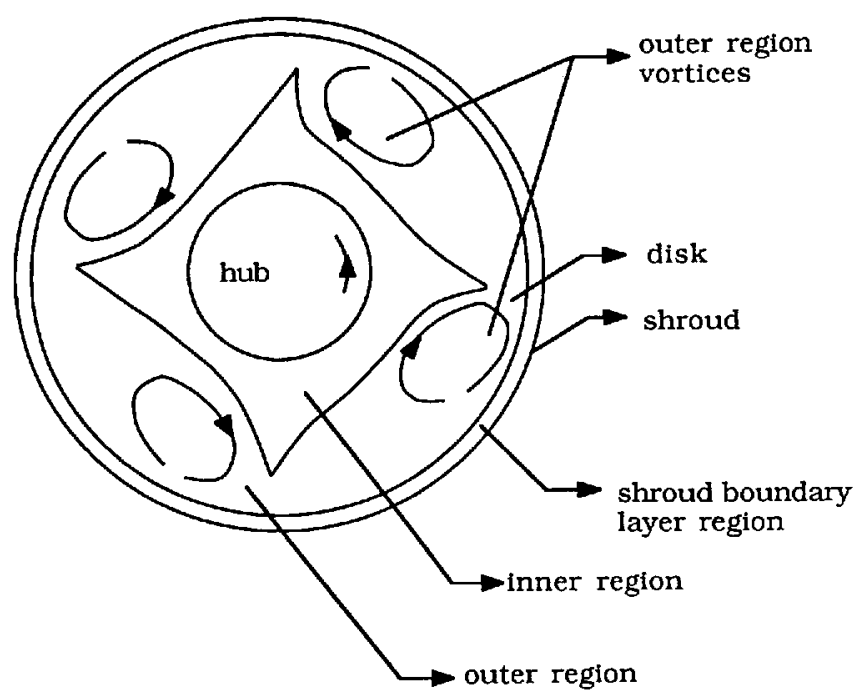

Fig. 5: Three different regions for flow between two corotating disks.

The outer region extends from the inner region to the outer radius of the disk. The flow is more turbulent and is dominated by large vortical structures. The precession rate of the vortical structures was estimated to be $75 \%$ of the disk rotation speed under the test condition. It is the outer region vortices that give the polygon shape to the inner region. The number of vortices and the stability of the region are determined by the Ekman 
number and disk-disk spacing. Between the outer radius of the disk and the shroud is the shroud boundary layer region, where the flow is three dimensional and the most turbulent. Toroidal vortex pair exists in this region, and has been hypothesized to play a major role in the formation of the vortices in the outer region.

The above discussions apply to a simplified geometry only. The presence of cooling flow and arms will modify the flow but main features still exist. This is especially true for the inner region. One can refer to Abrahamson et al. [7] for details.

Flow unsteadiness and turbulence make the numerical simulation difficult. Ananth [6] simulated the flow in corotating disks assuming a laminar steady flow and rotational symmetry. Although the simulation was not perfect, the qualitative features of the flow was captured.

Ananth [6] assumed there were no cooling flow in his numerical simulation for the flow in corotating disks. The calculation domain consists of $62(r) \times 6(\theta) \times 22(z)$ grids. It covers the area from the outer radius of the disk hub to the shroud in the radial direction, and half of the disk-disk spacing and half the disk thickness in the $z$ direction. The domain is 45 degree and evenly divided in the $\theta$ plane. Parametric studies have been made by Ananth [6] and one of his study case is re-calculated and shown here. Figures 6 (a)-(d) are the simulated results, where the streamlines and velocity profiles are shown under the following conditions - Re: 150.000 , disk radius: $8^{\prime \prime}$, hub radius: $4.8 \mathrm{~cm}$, disk-disk spacing: $0.635 \mathrm{~cm}$, disk-shroud spacing: $0.32 \mathrm{~cm}$, disk thickness: $0.32 \mathrm{~cm}$, medium: air at $20^{\circ} \mathrm{C}$. In Figure 6(a), the normalized streamlines with values ranging from 0.1 to 1.0 in the increment of 0.1 at the $\theta$ plane are plotted. The stream functions are calculated from the radial and axial velocities and are normalized with respect to the maximum value. It is seen that the fluid is pumped outward close to the disk surfaces because of viscous and centrifugal forces. To conserve mass, there is an inflow in the mid-plane. The size of the recirculation region increases with respect to the Reynolds number. The counter rotating vortices in the shroud region are present and their sizes are squeezed down with increasing Reynolds number.

Figure 6(b) shows the constant dimensionless angular velocity lines from values 1.0 to 0.1 with an increment of 0.1 . Lines with values equal to 1.0 correspond to the disk surface, and lines with values equal to 0.0 correspond to the stationary wall. The angular velocities are normalized with respect to the disk angular speed. The corresponding profiles with respect to the dimensionless radius at different dimensionless axial distances (normalizing distance is the disk-disk half spacing) measured from the bottom disk is shown in Figure 6(c). The dimensionless radius is the ratio of the radial distance from the hub to the radial distance between the hub and casing. In both figures, it is seen that large portion of the fluid is in solid body rotation, beginning from the hub to the half way between the tip of the disk and hub. Beyond that region, the fluid slows down gradually to the dimensionless angular velocity value of 0.7 at a point close to the disk tip, and sharply to zero at the casing.

Figure 6(d) shows the dimensionless radial velocity profiles (in percentage) with respect to the dimensionless axial length at different dimensionless radii. The dimensionless radius is now defined as the ratio of the radial distance from the hub to the radial distance between the hub and the tip of the disk (or $r / r_{2}$ in the figure). The normalizing factor for the radial velocity profiles is the local disk linear speed. It is seen that the radial component is negligibly small in the disk-disk region. For exam- ple, at $r / r_{2}=0.6$, the maximum radial velocity is only $2 \%$ of the local disk linear speed at the dimensionless axial distance of 0.4 (or $z=1270 \mu \mathrm{m}$ ). Below $r / r_{2}=0.4$, there are virtually no radial and axial velocity components throughout the entire diskdisk spacing. As will be shown later, this gives rise to a very slow radial transport of wear particles in the corotating disks.
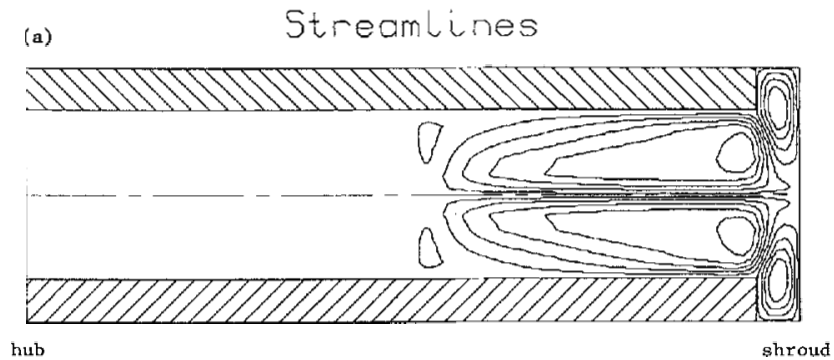

(b) Lines of const. angular velocity
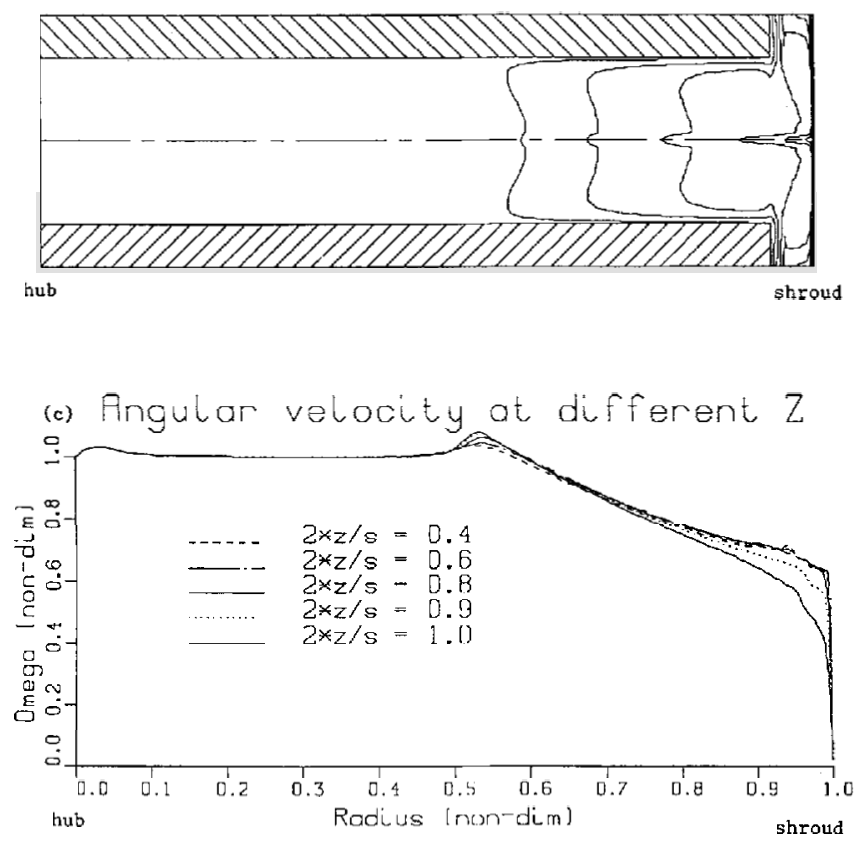

(d) Radial velocity at differont radii

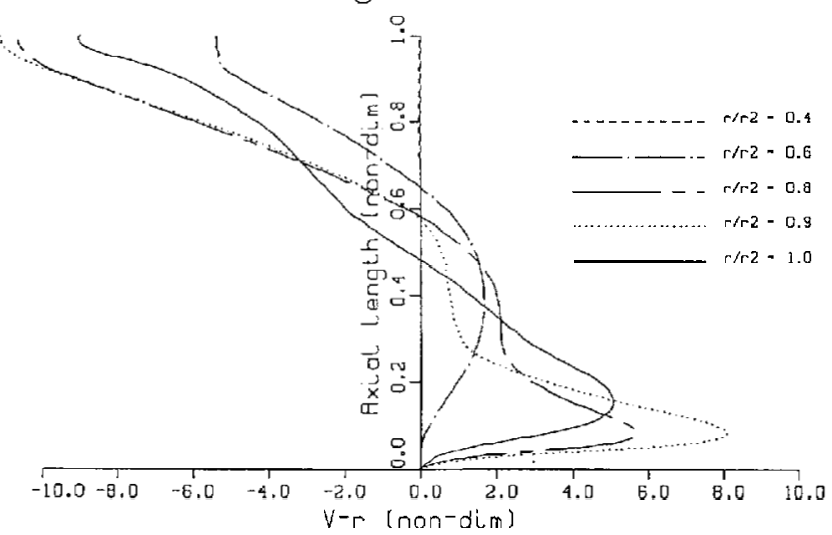

Fig. 6: Simulated flow field between two corotating disks, without cooling air flow. (a) streamlines in constant $\theta$ plane (b) lines of constant angular velocity (c) angular velocities at different $z(d)$ radial velocities at different radii. 


\subsection{Present Numerical Solutions}

Present fluid flow solutions employ a three dimensional finite difference program which is an extension of the original two dimensional finite difference scheme described in details in Patankar [8]. The present program uses the SIMPLER (SemiImplicit Method for Pressure Linked Equations Revised) algorithm for discretizing the momentum and continuity equations. For the solution of the discretized equations, the regular

(a) Streamlines

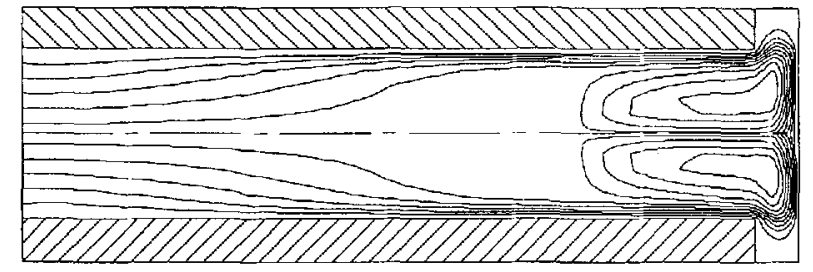

hub

(b) Lines of const. angular velccity
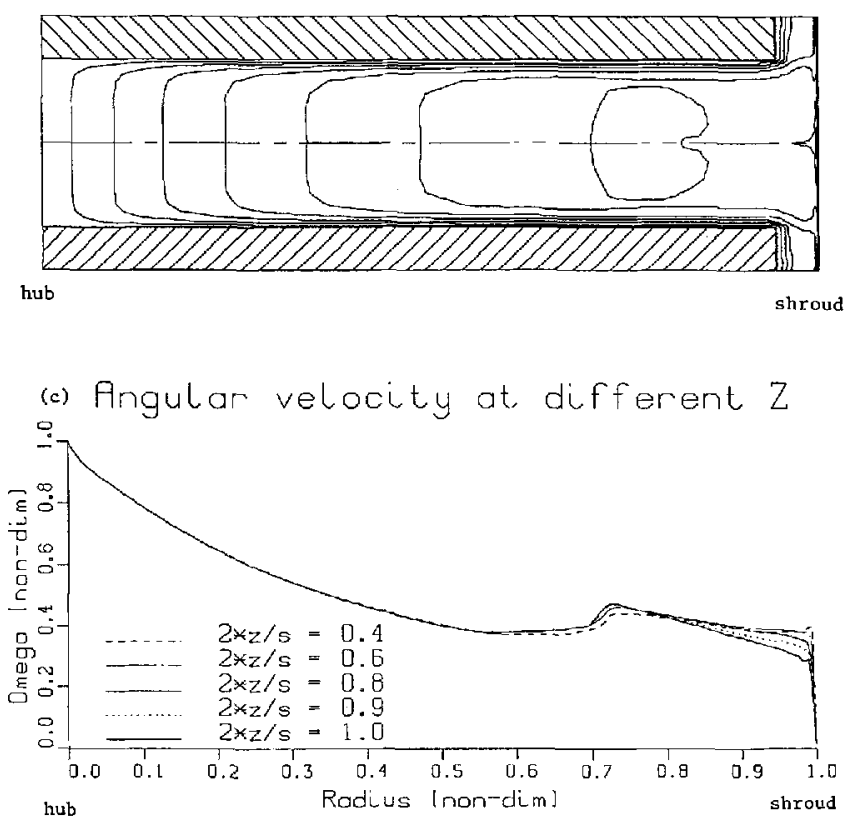

(d) Radial velocity at different radii

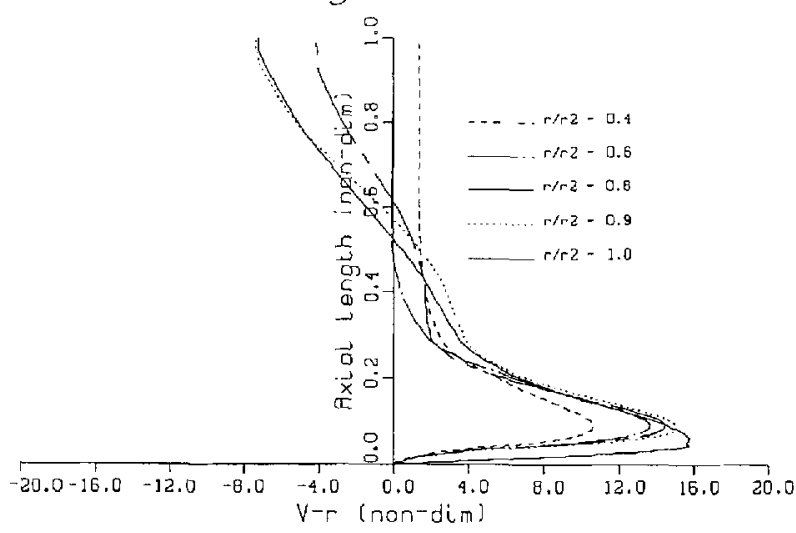

Fig. 7: Simulated flow field between two corotating disks, with cooling air flow $(120 \mathrm{slpm})$. (a) streamlines in constant $\theta$ plane (b) lines of constant angular velocity (c) angular velocities at different $z$ (d) radial velocities at different radii.
TDMA is used in both $r$ and $z$ directions, the circular TDMA is employed in the $\theta$ direction. Additional details can be seen in Ananth [6].

The present simulation takes into account the cooling air flow on the flow field and particle transport. The cooling air flow is assumed to be injected uniformly from the disk hub toward the disk spacing, and exhausted through the shroud region. Figures 7 (a) - (d) are the simulated results for the flow rate of 120 slpm per pair of disks. Other parameters are the same as before.

When a cooling flow rate of 120 slpm is added, the resulting flow field is modified. The recirculation region is reduced in the disk-disk region and it disappears in the disk-shroud region (Figure 7(a)). The streamlines show a strong radial flow from the hub toward the shroud. The inner region is completely gone, and the dimensionless angular velocity drops monotonically from the hub until the dimensionless radius reaches 0.5 (Figures 7 (b) and 7(c)). The radial velocity component (Figure $7(d)$ ) is increased and peaks at the dimensionless axial distance of 0.1 . At $r / r_{2}=0.4$ and 0.6 , the peak dimensionless values are now $10.5 \%$ and $13.5 \%$ respectively. The axial velocity components also increase correspondingly. Flow fields for other cooling flow rates of 12,24 and 48 slpm have also been calculated and the results for the case of $12 \mathrm{slpm}$ can be seen in Tsai [5]. The calculated flow fields will be used to examine the effectiveness in wear particle transport away from its generation spot.

\section{Wear Particle Transport and Deposition}

\subsection{Governing Equations and Numerical Methods}

To simulate wear particle motion, the particle equations of motion have to be integrated numerically. The numerical method to find particle trajectories has been documented in Ananth [6], and Tsai and $P u i$ [9]. The equations in the $r-\theta-z$ coordinate, which have been derived by Ananth [6], are re-written below incorporating the effects of external forces in the $z$ direction

$\ddot{r}=\frac{C_{\mathrm{d}} R e_{\mathrm{p}}}{24} \frac{18 \mu}{C \rho_{\mathrm{p}} D_{\mathrm{p}}^{2}}\left(V_{\mathrm{r}}-\ddot{r}\right)+r \dot{\theta}^{2}$

$\ddot{\theta}=\frac{C_{\mathrm{d}} R e_{\mathrm{p}}}{24} \frac{18 \mu}{C \rho_{\mathrm{p}} D_{\mathrm{p}}^{2}}\left(\frac{V_{\theta}}{r}-\dot{\theta}\right)-\frac{2}{r} \dot{r} \dot{\theta}$

$\ddot{z}=\frac{C_{\mathrm{d}} R e_{\mathrm{p}}}{24} \frac{18 \mu}{C \rho_{\mathrm{p}} D_{\mathrm{p}}^{2}}\left(V_{z}-\dot{z}\right)-\frac{F_{\mathrm{e}}}{m}$

where $C_{\mathrm{d}}$ is the drag coefficient, $R e_{\mathrm{p}}$ is the particle Reynolds number, $\mu$ is the dynamic viscosity, $C$ is the slip correction factor, $\rho_{\mathrm{p}}$ is the particle density, $D_{\mathrm{p}}$ is the particle diameter and $m$ is the particle mass. $V_{\Gamma}, V_{\theta}, V_{\mathrm{z}}$ are the fluid velocities in $r, \theta$ and $z$ directions respectively. External forces $F_{\mathrm{e}}$ in the $z$ direction include gravitational $\left(F_{\mathrm{g}}\right)$, Coulombic $\left(F_{\mathrm{c}}\right)$ and image $\left(F_{\mathrm{i}}\right)$ forces. Each component can be calculated as: $F_{\mathrm{g}}=m g$, $F_{\mathrm{c}}=n e E$ and $F_{\mathrm{i}}=n^{2} e^{2} /\left(4 z^{2}\right) f(\varepsilon)$, where $n$ is the number of elementary units of charge, $e$ is the charge of an electron, $z$ is the distance between the particle and the surface. Dielectric conductivity factor $f(\varepsilon)$ is equal to $(\varepsilon-1) /(\varepsilon+1)$ with $\varepsilon$ the dielectric constant. In the current simulation, the particle is assumed to be conductive, hence $\varepsilon=\infty$ and $f(\varepsilon)=1.0$. 
The effect of Brownian diffusion is also taken into consideration. If the elapsed time is $t_{\mathrm{t}}$ after the generation of a particle, the rms diffusional displacement in the vertical direction from the mean trajectory of a particle can be calculated to be $\sqrt{2 D t_{\mathrm{t}}}$, where $D$ is the diffusivity of the particle. If the particle comes close to the surface within such a distance, the particle has a finite possibility $(16 \%)$ to be intercepted by the surface. In the current simulation, it is assumed that particle deposition occurs when it approaches the surface within a distance less than $\sqrt{2 D t_{\mathrm{t}}}$.

Considering the effect of external force is important since wear particles will be stopped initially very close to the disk surface as shown in Figure 4. A wear particle has been found to carry electric charge upon its generation by $P u i$ et al. [10], and its charge level has been measured. This is shown in Figure 8 for wear particles generated from an oxide medium disk. The best fit through the data points indicates the number of elementary units of charge is 17 times the aerodynamic diameter. It is this relationship that will be used to investigate the electrostatic effect on wear particle transport and deposition.

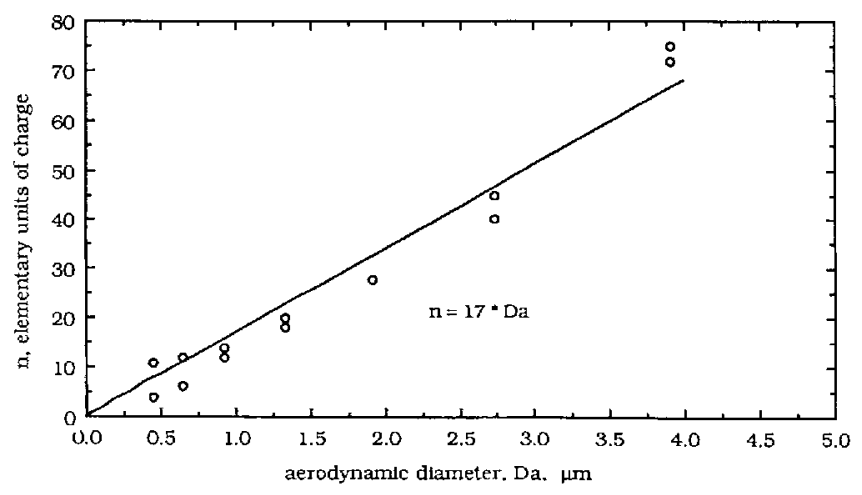

Fig. 8: Electric charge carried by airborne wear particles versus aerodynamic diameter.

Figure 9 shows the migration distances per second due to different external forces. It is seen that except the convective transport by fluid, the gravitational migration is the most important transport mechanism for particles greater than $1.0 \mu \mathrm{m}$. For submicron particles, the coulombic force can be important than the diffusional transport when the electric field is greater than $10 \mathrm{volt} / \mathrm{cm}$. The image force is relatively unimportant except when the particle is nearly in contact with the surface. For comparison, the axial fluid velocity is shown in the same figure as dash line at a vertical location whose distance is equal to one particle diameter. In case of a single rotating disk, the downward axial fluid velocity can be approximated to be $0.17 z \omega$, where $z$ is the vertical distance from the disk surface and $w$ is the disk rotating speed. It is seen that although the axial fluid velocity is small close to the disk surface, it is still very important for submicron particle transport and deposition.

The computer program assumes an initial particle location and speed, uses the previously obtained flow field data, and integrates the equations of motion by the Runge-Kutta method. The initial particle velocity can be calculated using the previous model. However, as Figure 3 shows, the vertical component in "drag mode" is very small compared to the horizontal component. If the calculated initial velocities were used, it is foreseen that particles will be moving right on the surface since there is no upward axial velocity in most cases. In the following ana-

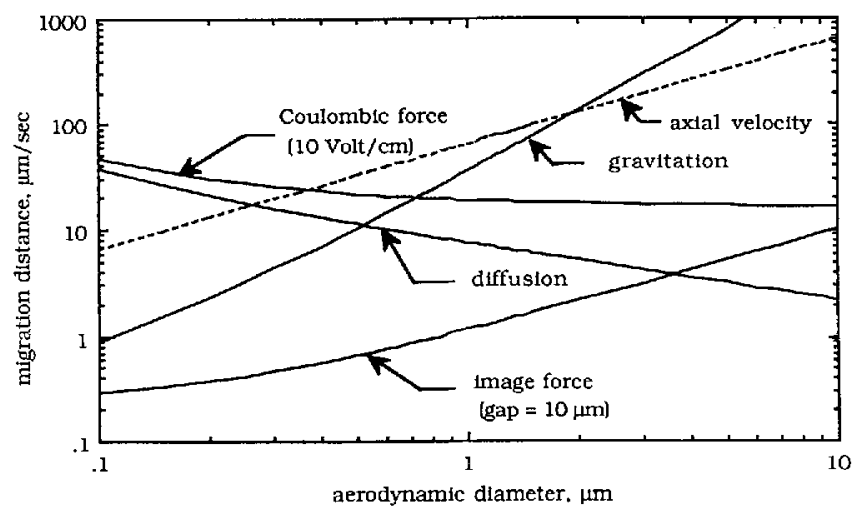

Fig. 9: Migration distance per second due to different external forces.

lysis, the "worse" scenario is used. It is assumed that particles are shooting at $\mathbf{4 5}$ degree from the disk surface and the initial particle velocity is the same as the local disk linear velocity.

\subsection{Simulated Wear Particle Trajectories}

Figures 10 and 11 show the trajectories of 1.0 and $4.0 \mu \mathrm{m}$ particles for the cases without and with cooling flow including all but Coulombic force. When the cooling flow is not present, a $1.0 \mu \mathrm{m}$ particle keeps on rotating (Figure $10(\mathrm{a})$ ) until it finally deposits at a radial distance less than $1.0 \mathrm{~cm}$ from the original position (note the program stops after 10 revolutions). For a $4.0 \mu \mathrm{m}$ particle (Figure 10(b)), it migrates toward the diskshroud region and revolves there for very long time. With the 12 slpm cooling flow rate, the $1.0 \mu \mathrm{m}$ particle (Figure 11 (a)) still deposits on the disk surface but much remote $(4.3 \mathrm{~cm})$ from the original radial position where the particle is first generated. For a $4.0 \mu \mathrm{m}$ particle (Figure 11 (b)), it migrates out of the disk-disk region in two turns. Thus, adding cooling air flow results in more rapid transport of wear particles away from the disk-disk spacing. It is expected that disk crashes can be minimized because of less deposited particles on the disk surface.

The electric field on the disk surface will influence wear particle deposition. Figure 12 shows the effect of electric field $(0,10$ and $50 \mathrm{volt} / \mathrm{cm}$ ) on the critical radial distance from the disk hub. The critical radial distance is defined such that a wear particle will deposit on the disk surface when it is generated within a radial distance smaller than it. When there is no electric field, the generated wear particle will not deposit on the disk surface nearly at all radial generation sites when it is greater than $1.0 \mu \mathrm{m}$ in aerodynamic diameter. It is clear from the figure that increasing the electric field will enhance wear particle deposition. This is undesirable in a disk drive.

To investigate the effect of the cooling flow rate on wear particle removal, Figure 13 shows the critical radial distance measured from the disk hub versus wear particle aerodynamic diameter. It is seen that no matter how high the cooling flow rate is, entire removal of the generated, submicron wear particles is not possible at all radial generation positions. This is because a boundary layer is formed as soon as the cooling flow is added. However, when the flow rate is too small, the radial component is too small to transport even particles greater than $1.0 \mu \mathrm{m}$ away from the generation site. The optimum flow rate is determined as 12 slpm in the case of $8^{\prime \prime}$ disk with the Reynolds number of 150.000. At a smaller Reynolds number, it is foreseen that this cooling flow rate will be more effective in wear particle removal. 


\section{Conclusions}

This study is concerned with the understanding of the fundamental wear particle transport and deposition problems. Results of analytical and numerical analyses have shown that wear particles are emitting from the trailing edge of the slider at a very small vertical speed. As a result, large or small wear particles are flying very close to the disk surface. Experimental comparison of wear particle concentrations using two different sampling methods has suggested that wear particles are indeed flying very close to the disk surface. Particles can then deposit on the disk surface easily causing further damage to the disk drive.

In the case of corotating disks, large or small wear particles are entrapped and deposit inside disk drives when the cooling flow is not present. For submicron particles, they almost deposit on the same radial position as its first generation site and are expected to cause disk to crash more often as they accumulate to a bigger size. Cooling air flow from the disk hub is effective in removing wear particles greater than $1.0 \mu \mathrm{m}$. However, too high a cooling flow rate from the disk hub is not necessary since complete removal of all submicron particles from the disk drive is not possible due to the boundary layer effect. The parametric study has indicated a cooling air flow rate of 12 slpm is op- timum for a $8^{\prime \prime}$ disk drive with the Reynolds number of 150.000 . Electrostatic effect enhances wear particle deposition in the case of corotating disks. To reduce undesirable wear particle deposition, the electric field in the disk drive must be eliminated.

\section{Acknowledgement}

This research was supported partially by the Graduate School of the University of Minnesota through its Doctoral Dissertation Fellowship during the academic year 1988-1989. Supercomputer time for fluid flow and particle simulations was granted by the Minnesota Supercomputer Institute. Most of the financial support has come from the Particulate Contamination Control Research Consortium at the University of Minnesota. Members of the consortium include Air Products and Chemicals, Inc., Airco/The BOC Group, Applied Materials, Donaldson Co., FSI International, GM/Delco Electronics, IBM Corp., Millipore Corp., Nupro Co., TSI Inc. The supports of the Graduate School, the Minnesota Supercomputer Institute and the consortium members are gratefully acknowledged.

(a)

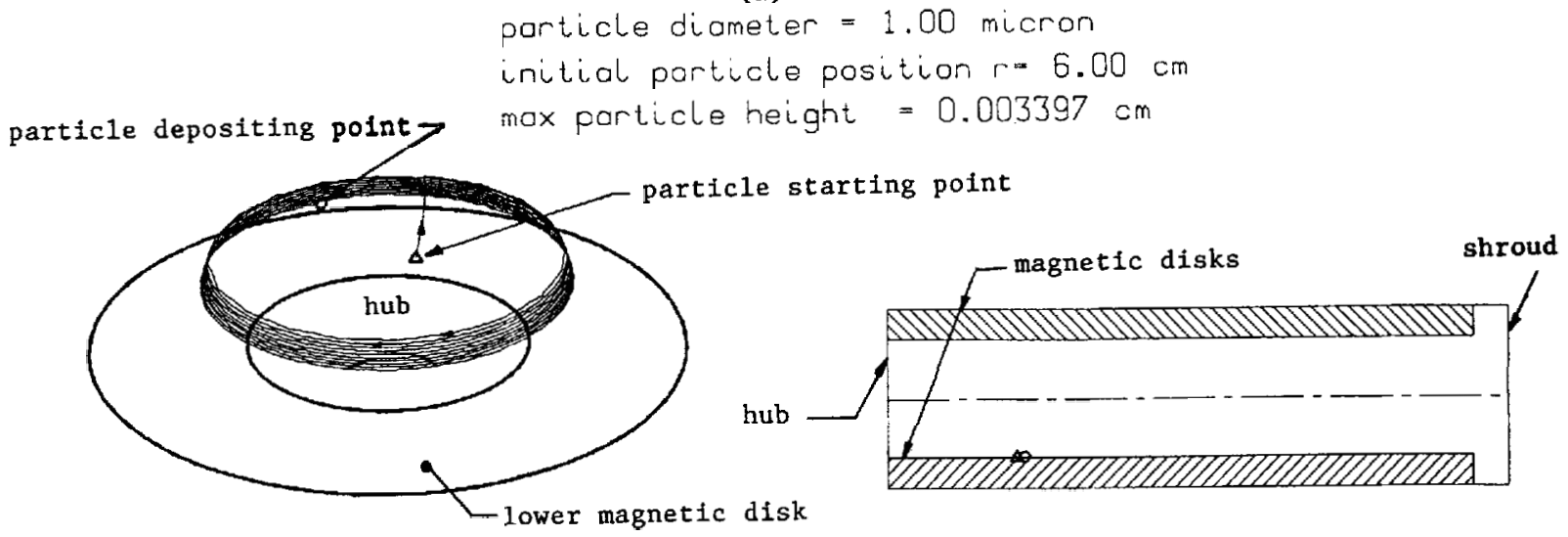

(b)

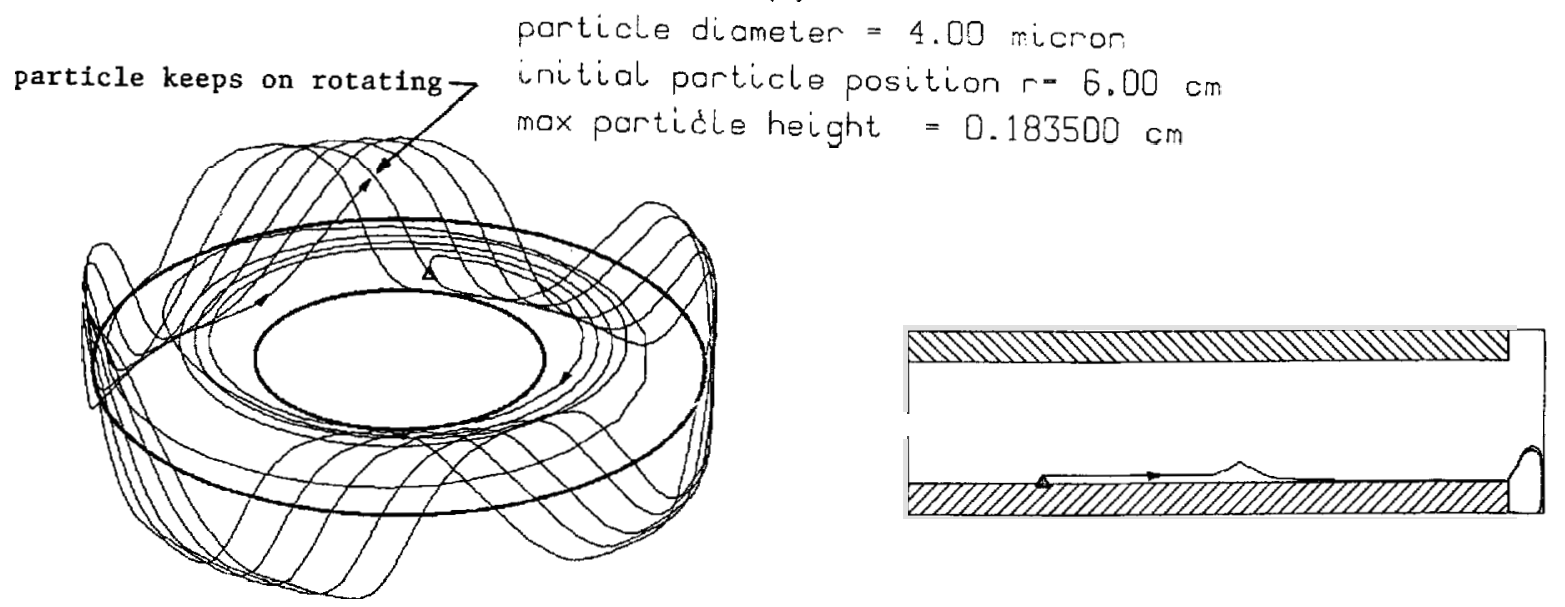

Fig. 10: Wear particle trajectories in corotating disks, without cooling air flow, (a) $D_{\mathrm{a}}=1 \mu \mathrm{m}$ (b) $D_{\mathrm{a}}=4 \mu \mathrm{m}$. 
(a)

particle diameter $=1.00$ micron

initial porticle position $r=6.00 \mathrm{~cm}$

max porticle height $=0.110691 \mathrm{~cm}$
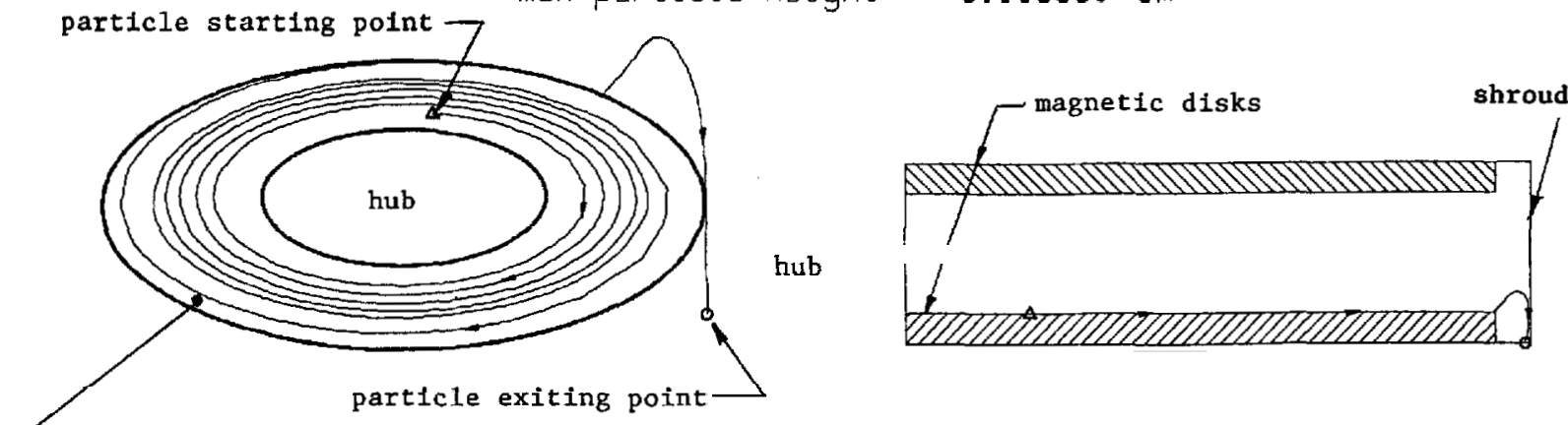

$\angle$ lower magnetic disk

(b)

particle diameter $=4.00$ micron

initial particle position $r=6.00 \mathrm{~cm}$

max porticle height $=0.109944 \mathrm{~cm}$
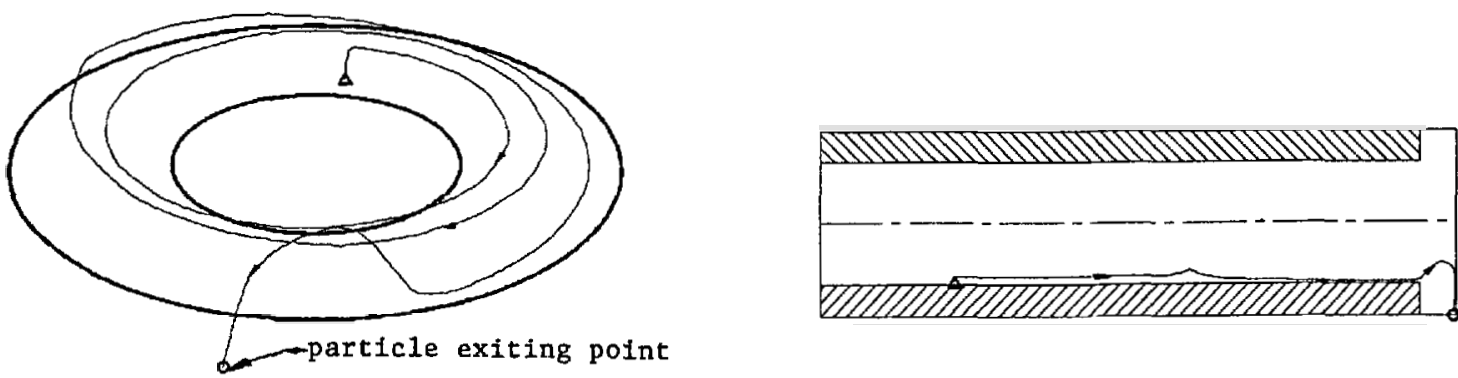

Fig. 11: Wear particle trajectories in corotating disks, with cooling air flow (12 slpm), (a) $D_{\mathrm{a}}=1 \mu \mathrm{m}$ (b) $D_{\mathrm{a}}=4 \mu \mathrm{m}$.

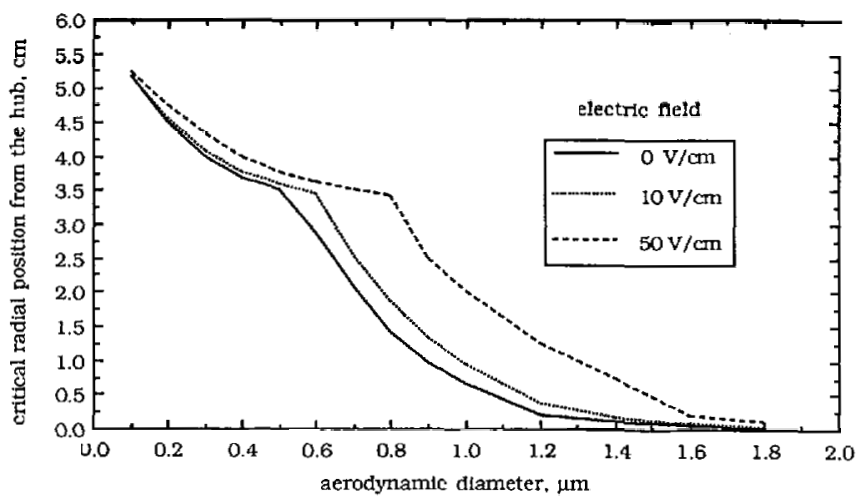

Fig. 12: Effect of electric field on the critical radial starting position from the disk hub.

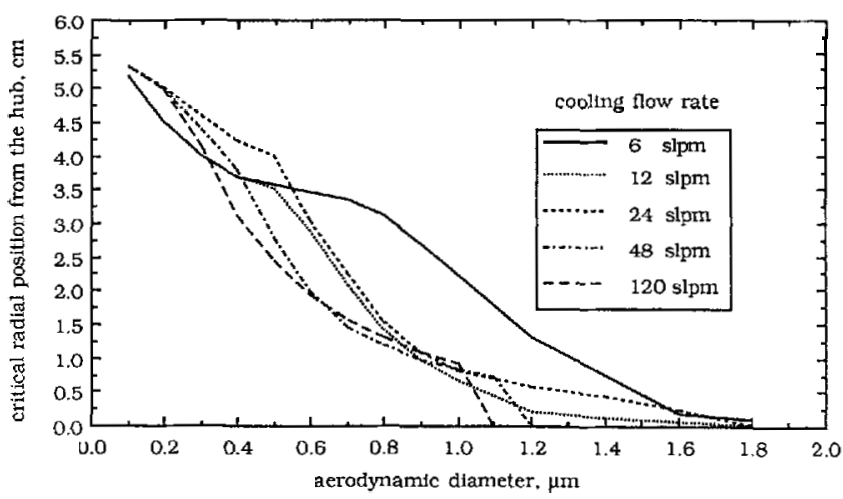

Fig. 13: Effect of cooling flow rate on the critical radial starting position from the disk hub.

\section{Symbols and Abbreviations}

$A_{\mathrm{n}} \quad$ nominal contact area

$b \quad$ flake-shaped particle width

$b^{\prime} \quad$ equal area radius of a flake-shaped particle

$C_{\mathrm{d}} \quad$ drag coefficient

C slip correction factor
$C_{\mathrm{D}_{\mathrm{ev}}} \quad$ slip correction factor for equal volume diameter of the flake

$D \quad$ particle diffusion coefficient

$D_{\mathrm{a}} \quad$ particle aerodynamic diameter

$D_{\mathrm{p}} \quad$ particle geometric diameter

$e \quad$ charge of an electron

E Young's Modulus 
$E_{\mathrm{kv}} \quad$ kinetic energy in vertical direction

$f(\varepsilon)$ dielectric conductivity factor

$F_{\mathfrak{c}} \quad$ Coulombic force

$F_{\mathrm{g}} \quad$ gravitational force

$F_{\mathrm{i}} \quad$ image force

$F_{\text {r }} \quad$ real contact load

$K^{\prime} \quad$ shape factor for a flake

$L \quad$ flake-shaped particle length

$n$ number of elementary units of charge

$r_{2} \quad$ disk radius

Re Reynolds number based on the disk tip speed

$r \quad r$ coordinate

$\dot{r}$ first time derivative of $r$

$\ddot{r} \quad$ second time derivative of $r$

$t \quad$ flake-shape particle thickness

$t_{\mathrm{t}}$ time

$V_{\mathrm{d}} \quad$ disk linear speed

$V_{\mathrm{h}} \quad$ horizontal particle velocity component

$V_{\mathrm{r}} \quad$ fluid velocity in $r$ direction

$V_{\mathrm{v}} \quad$ vertical particle velocity component

$V_{\mathrm{z}} \quad$ fluid velocity in $z$ direction

$V_{\theta} \quad$ fluid velocity in $\theta$ direction

$z \quad z$ coordinate

$\dot{z}$ first time derivative of $z$

$\ddot{z} \quad$ second time derivative of $z$

$\varepsilon \quad$ dielectric constant

$\mu_{\mathrm{f}} \quad$ coefficient of friction $\mu \quad$ dynamic viscosity of air

$\rho_{\mathrm{p}} \quad$ particle density

$\theta \quad \theta$ coordinate

$\dot{\theta}$ first time derivative of $\theta$

$\ddot{\theta} \quad$ second time derivative of $\theta$

\section{References}

[1] I. Croll: ASLE Special Publication, SP-16. 2 (1984) 1-6.

[2] Porter Report: 1989 Disk/Trend Report (1989) Disk/Trend Inc.

[3] M. L. Lesser, J. W. Haanstra: IBM J. Res. Dev. 1 (1957) 17.

[4] C.J. Tsai, D. Y. H. Pui, B. Y. H. Liu: (in press) Aerosol Sci. Tech. June, Vol. 6, 1991.

[5] C.-J, Tsai: A Particle Phenomena in Computer Disk Drives. Ph. D. thesis. Mechanical Engineering Department, University of Minnesota 1990.

[6] G. P. Ananth: Experimental and Numerical Investigations of Particulate Contamination in Disk Drives. Ph. D. thesis, Mechanical Engineering Department, University of Minnesota 1988.

[7] S. D. Abrahamson, D. J. Koga, J. K. Eaton: Report MD-50 Thermosciences Division. Department of Mechanical Engineering, Stanford University, Stanford, California 1988.

[8] S. V. Patankar: Numerical Heat Transfer and Fluid Flow. Hemisphere 1980.

[9] C. J. Tsai, D. Y. H. Pui: Numerical Study of Particle Deposition in Bends of Circular Cross Section. Aerosol Sci. Tech, 12 (1990) 813-831.

[10] D. Y. H. Pui, C.J. Tsai, B. Y. H. Liu: Charge Level on Aerosol Particles. 34th annual technical meeting, Institute of Environmental Sciences, King of Prussia, Pennsylvania, May 2-6, 1988. 